\title{
NLRP1 and NLRP3 inflammasomes as a new approach to skin carcinogenesis (Review)
}

\author{
MAGDALENA CIĄŻYŃSKA $^{1 *}$, IGOR A. BEDNARSKI ${ }^{2 *}$, KAROLINA WÓDZ $^{3^{*}}$, \\ JOANNA NARBUTT $^{2 *}$ and ALEKSANDRA LESIAK ${ }^{2 *}$
}

\begin{abstract}
${ }^{1}$ Department of Proliferative Diseases, Nicolaus Copernicus Multidisciplinary Centre for Oncology and Traumatology, Lodz 93-513; ${ }^{2}$ Department of Dermatology, Pediatric Dermatology and Dermatological Oncology, Medical University of Lodz, Lodz 91-347; ${ }^{3}$ Laboratory of Molecular Biology, VET-LAB, Brudzew 62-720, Poland
\end{abstract}

Received August 6, 2019; Accepted October 16, 2019

DOI: $10.3892 / \mathrm{ol} .2020 .11284$

\begin{abstract}
Inflammasomes are key innate immune system receptors that detect pathogenic endo- and exogenous stressors like microorganisms or ultraviolet radiation (UVR) which activate the highly proinflammatory cytokines interleukin- $1 \beta$ and interleukin-18. Inflammasomes are not only involved in inflammation, but also in carcinogenesis and tumor progression. Due to the dynamic increase in non-melanoma skin cancers (NMSC), it has become necessary to determine how UVR, which plays a key role in NMSC development, can regulate the structure and function of inflammasomes. In the present study, the regulatory mechanisms of NOD-Like Receptor Family Pyrin Domain Containing 1 and 3 inflammasome activation as well as an effective inflammasome-mediated immune response after UVR exposition are discussed. The differences and similarities between these molecular complexes that monitor cellular health, inflammation, and skin carcinogenesis are also highlighted. Despite numerous scientific data, more studies are still required to better understand the biology of inflammasomes in skin cancer development and to explore their therapeutic potential.
\end{abstract}

\section{Contents}

1. Introduction

2. Inflammasomes: Mechanism of function depends on structure

3. Influence of UVR on the activation of an inflammasome

4. NLRP3 and skin carcinogenesis

Correspondence to: Dr Magdalena Ciążyńska, Department of Proliferative Diseases, Nicolaus Copernicus Multidisciplinary Centre for Oncology and Traumatology, ul. Pabianicka 62, Lodz 93-513, Poland E-mail: ciazynska.magdalena@gmail.com

*Contributed equally

Key words: inflammasome, NLRP1, NLRP3, skin carcinogenesis, UVR
5. NLRP1 and skin carcinogenesis

6. Therapies that target inflammasomes

7. Conclusions

\section{Introduction}

An increasing body of evidence indicates that inflammation is one of the hallmarks of carcinogenesis, allowing the growth and metastasis of tumors (1). The role of inflammation is two-fold: On one hand, the tumor and antigens present on the surface of cells can stimulate effector cell recruitment of the innate immune system, resulting in the destruction of diseased tissue; on the other hand, mere stimulation of the immune response is associated with the development of inflammation, which can stimulate the growth of a tumor through the production of proinflammatory cytokines, thus creating a feedback loop (2). A special role in this process is played by interleukin $1 \beta$ (IL-1 $\beta$ ), which promotes tumor progression, neoangiogenesis, and metastasis (3). Activation of IL-1 $\beta$ depends on the function of the proteolytic enzyme, caspase-1, which is strictly regulated by multiprotein complexes called inflammasomes.

Many endo- and exogenous factors can be involved in the process of inflammasome activation. However, due to a dynamic increase in non-melanoma skin cancers (NMSC) in the Caucasian population, it has become necessary to determine how ultraviolet radiation (UVR), which plays a key role in NMSC development, can regulate the structure and function of inflammasomes. This review presents a current overview of the inflammasome activation and regulation of NLRP3 (NOD-Like Receptor Family Pyrin Domain Containing 3) and NLRP1 (NOD-Like Receptor Family Pyrin Domain Containing 1). Recent findings about the inflammasome-mediated immune response after UVR exposition are also analyzed, and a comparison of the structures and the regulation of NLRP3 and NLRP1 inflammasomes is made.

\section{Inflammasomes: Mechanism of function depends on structure}

The immune system protects the human body against various infections and tissue damage through the development 
of a broad range of proteins that form multimeric active inflammasomes. As part of an innate immune response, inflammasomes initiate inflammation in response to cellular stress induced by non-infective agents (e.g., reactive oxygen species and lysosomal enzymes) commonly known as DAMPs (Danger Associated Molecular Patterns) as well as pathogenic microbes known as PAMPs (Pathogen Associated Molecular Patterns) (4).

The function of an inflammasome depends on its structure. In general, the core of an inflammasome consists of nucleotide-binding oligomerization domain-like receptors (NOD-like receptors, NLRs). The binding of NLRP to ASC (apoptosis associated with Speck-like protein) and procaspase-1 results in the formation of a functional inflammasome and activates caspase- 1 by autocatalytic cleavage (5). Active caspase- 1 further processes pro-IL-1 $\beta$ and pro-IL-18 into their mature and bioactive forms (IL-1 $\beta$ and IL-18), which induces the secretion of subsequent factors that activate the inflammatory process. The ASC protein is composed of two death-fold domains: one pyrin domain (PYD) and one caspase activation and recruitment domain (CARD). The PYD and CARD domains mediate homotypic interactions with other proteins containing a PYD or a CARD domain. The NLR family comprises 22 proteins that are present in humans; however, not all of them can form an inflammasome. NLRP1, NLRP2, NLRP3, NLRP6, NLRP7, NLRC4 (NLR family CARD domain-containing protein 4), NLRP12, and AIM2 (absent in Melanoma 2) have been reported to initiate the formation of inflammasomes (6).

In the last few years, important advances in the understanding of NLRP1 and NLRP3 biology have been achieved. In principle, NLR proteins, except for NLRP1, have a tripartite domain organization which may contain PYD at the $\mathrm{N}$-terminus to bind ASC; nucleotide binding and an oligomerization domain (NACHT) in the middle which are responsible for the oligomerization of the inflammasome; and leucine-rich repeats (LRRs) at the C-terminus which bind to PAMPs or DAMPs (Fig. 1) (5,7).

In addition to these three domains, NLRP1 contains a function-to-find domain (FIIND) and CARD. Moreover, the N-terminal pyrin domain (PYD) is also found in human NLRP1 and other non-rodent species but is absent in mice. The presence of two effector domains (PYD and CARD) has caused confusion regarding the role of each domain (Fig. 1A). For this reason, initially, the human NLRP1 was considered to be unique because it can activate caspase-1 through the CARD domain without recruiting ASC (5). The initial description of the NLRP1 inflammasome proposed also that the PYD is important as an adapter protein for binding ASC and then recruiting and activating caspase-1 (8). Currently, most studies indicate that under some conditions, caspase- 1 can connect directly to the C-terminal CARD domain, but only in murine NLRP1 (9-11). Interestingly, the direct activation of caspase-1 by NLRP1 with a bypassed ASC does not result in the proteolysis of caspase-1 which is still capable of producing mature IL-1 $\beta(12,13)$. Moreover, the initial description of the human NLRP1 inflammasome included NLRP1, ASC, caspase-1, and caspase-5. However, the exact role of caspase-5 in the canonical NLRP1 inflammasome activation still needs to be elucidated (14).
Surprisingly, Finger et al (15) demonstrated that human NLRP1 activity is dependent upon ASC which is associated with the C-terminal CARD domain. Furthermore, it has been shown that human NLRP1 activity is dependent upon autolytic cleavage in the FIIND domain (15). A recent study conducted by $\mathrm{Yu}$ et al (16) redefined our understanding of the role of the ASC protein in human NLRP1 function. It was concluded that the NLRP1 N-terminal domain (PYD in humans) is autoinhibitory, while the C-terminal cleavage fragment with the CARD domain engages an ASC dependent inflammasome.

NLRP inflammasomes are concerned with pyroptosis, a recently described pathway of programmed cell death. Activated caspase-1 results not only in processing and the release of inflammatory cytokines (IL-1 $\beta$, IL-18), but also in pyroptosis, which causes a loss of plasma membrane integrity (17).

A recent study indicated that during an invasive gram-negative bacterial infection, caspase-4/5 in humans and caspase-11 in mice bind to cytosolic lipopolysaccharide (LPS), promoting NLRP3 activation and forming a complex termed the non-canonical inflammasome $(18,19)$. The non-canonical inflammasome also activates pyroptosis, but only causes the processing of proinflammatory cytokines indirectly by activating the canonical inflammasome through a not well-defined mechanism (20). The NLRP1 inflammasome could also promote the activation of caspase- 1 and the subsequent activation and release of IL-1 $\beta$ as well as the initiation of pyroptosis (Fig. 2) (21).

\section{Influence of UVR on the activation of an inflammasome}

UVR represents one of the main environmental risks and stress factors for the skin. Excessive exposure to UVR can directly damage the DNA of dermal cells and, in addition, induces inflammation of the skin that is commonly termed sunburn. At a molecular level, this phenomenon is characterized by the activation of inflammasomes and stress pathways that include nuclear factor $(\mathrm{NF})-\kappa \mathrm{B}$. Both chronic and acute UVR exposures are potent complete carcinogens which initiate and promote cancer development. Physical and metabolic damage to the dermal cells caused by UVR exposition causes the release and accumulation of endogenous cellular components, extracellular DAMPs, which induce a 'sterile' inflammation. Different subtypes of NLR recognize specific DAMPs, such as IL-1 $\alpha$ and IL-33. These interleukins are two endogenous molecules that are perceived to be potent 'danger' signals that indicate the potential loss of epidermal barrier integrity (4). Normally, IL- $1 \alpha$ and IL-33 are present in the nuclei involved in transcription modulation and are released from cells under the influence of factors that induce-dependent on the inflammasome-unconventional secretion. The activation of an inflammasome is also associated with the unconventional secretion of HMGB-1 (high mobility group box protein 1), which is an evolutionarily conserved protein with a broad spectrum of actions. Inside cells, HMGB-1 is also found mainly in the cell nuclei, where it participates, e.g., in replication and DNA repair. However, when HMGB-1 is released into the extracellular space, it becomes a proin- 
A a mouse NLRP1 INFLAMMASOME

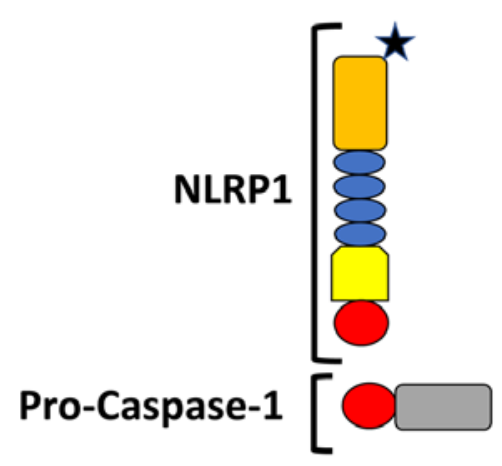

b human NLRP1 INFLAMMASOME

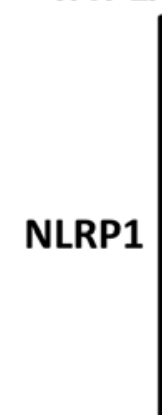

B NLRP3 INFLAMMASOME

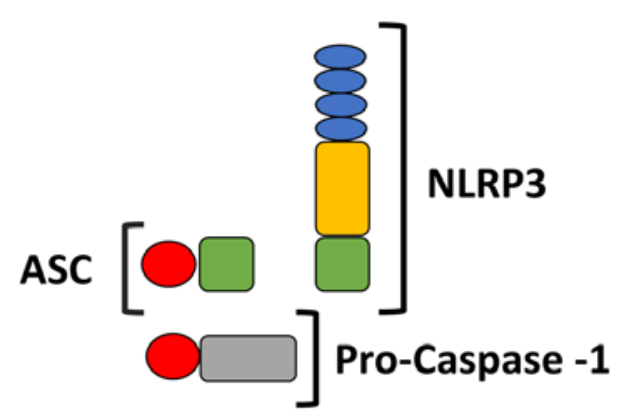
Pro-Caspase-1

Figure 1. Schematic representation of NLRP1 and NLRP3 inflammasomes. All NLRP1 inflammasomes are composed of NACHT, LRR, FIIND and CARD domains. Domain positions are indicated to scale. The presence of FIIND and CARD domains at the C-terminus makes NLRP1 stand out from all other members of the NLR family. However, the NLRP1 inflammasome is quite different in murine and human models. (A-a) Mouse NLRP1 inflammasome: In mice, there are three paralogs of NLRP1 (N1rp1a, -b, and -c) which contain an NR100 domain instead of the PYD found in humans. Mouse NLRP1 can activate caspase-1 directly and ASC (apoptosis associated with Speck-like protein) is not required. (A-b) Human NLRP1 inflammasome: Human NLRP1 contains the N-terminal PYD. Its activity is dependent upon ASC, which is associated with the C-terminal CARD domain. (B) NLRP3 inflammasome: The NLRP3 inflammasome consists of an NLRP3 protein, an ASC adaptor, and procaspase-1. Upon stimulation, NLRP3 and ASC adhere via their PYD domains, whereas ASC and caspase-1 adhere via their CARD domains. Therefore, NLRP3 forms an ASC-dependent inflammasome. NLRP1, NOD-like receptor family pyrin domain containing 1; NLRP3, NOD-like receptor family pyrin domain containing 3; NACHT, nucleotide-binding and oligomerization domain; LRR, leucine rich repeat; FIIND, function to find domain; PYD, pyrin domain; ASC, apoptosis associated with Speck-like protein.

flammatory cytokine which stimulates the formation of new blood vessels, enhances cell migration, and affects cell proliferation (Fig. 2).

UVR exposure stimulates keratinocytes to secrete an abundant amount of pro-inflammatory IL-1-family proteins, namely, IL-1 $\alpha$, IL-1 $\beta$, IL-18, and IL-33 (6). Under natural conditions, normal healthy skin contains low levels of the inactive precursor forms of IL-18 and IL-1 $\beta$ that require the presence of caspase-1 for maturation and secretion (4).

Previous research conducted in vitro indicated the occurrence of inflammasome-dependent secretion of mature IL-1 $\beta$ and IL-18 in human keratinocytes under the influence of UVB radiation (22-25). Hasegawa et al (23) indicated, for the first time, that the expression of the NLRP3 gene, like the production of IL- $1 \beta$ and other inflammatory mediators, including IL-1 $\alpha$, IL- 6 , TNF- $\alpha$, and PGE2 in keratinocytes, increases under intensity-dependent UVB radiation. In addition, immunofluorescence analysis research has indicated that not only UV-irradiated human keratinocytes, but also human epidermis, show elevated expression of NLRP3 (23). It is speculated that UVB-induced NLRP3 inflammasome activation is the connection between nuclear DNA damage responses and the production of various specific inflammatory mediators (23).
So far, it has been proven that some genetic changes in the NLRP family, that cause excessive activation of inflammasomes, predispose an individual to the formation of inflammatory skin lesions, such as psoriasis, atopic dermatitis, and vitiligo (26-28). As for other constitutively expressed NLRPs, mutations in NLRP1 are associated with skin diseases (28).

Based on the fact that keratinocytes show the constitutive expression of NLRP1, presumably, this NLR is likely to be the first responder to DAMP induced by UV-damage. Although NLRP1 is characterized by the activation of both caspase- 1 and caspase-5, which in human cells can activate IL-1 $\beta$ (8). However, the knockdown of only caspase-5 in keratinocytes does not significantly affect the secretion of IL-1 $\beta$. This finding suggests that inflammatory processes in keratinocytes caused by the action of UV-irradiation do not require caspase- 5 . This has been confirmed by the fact that various stimuli in a range of cell types can activate various NLR components (29). Active NLRP1 forms self-oligomers by binding to procaspase-1 and ASC (29). The mouse NLRP1 homologue, unlike the human one, lacks the PYD domain and is therefore unable to bind ASC. Bcl-2 (B-cell lymphoma 2) and Bcl-XL (B-cell lymphoma-extra-large) are anti-apoptotic proteins, which, in normal resting cells, 


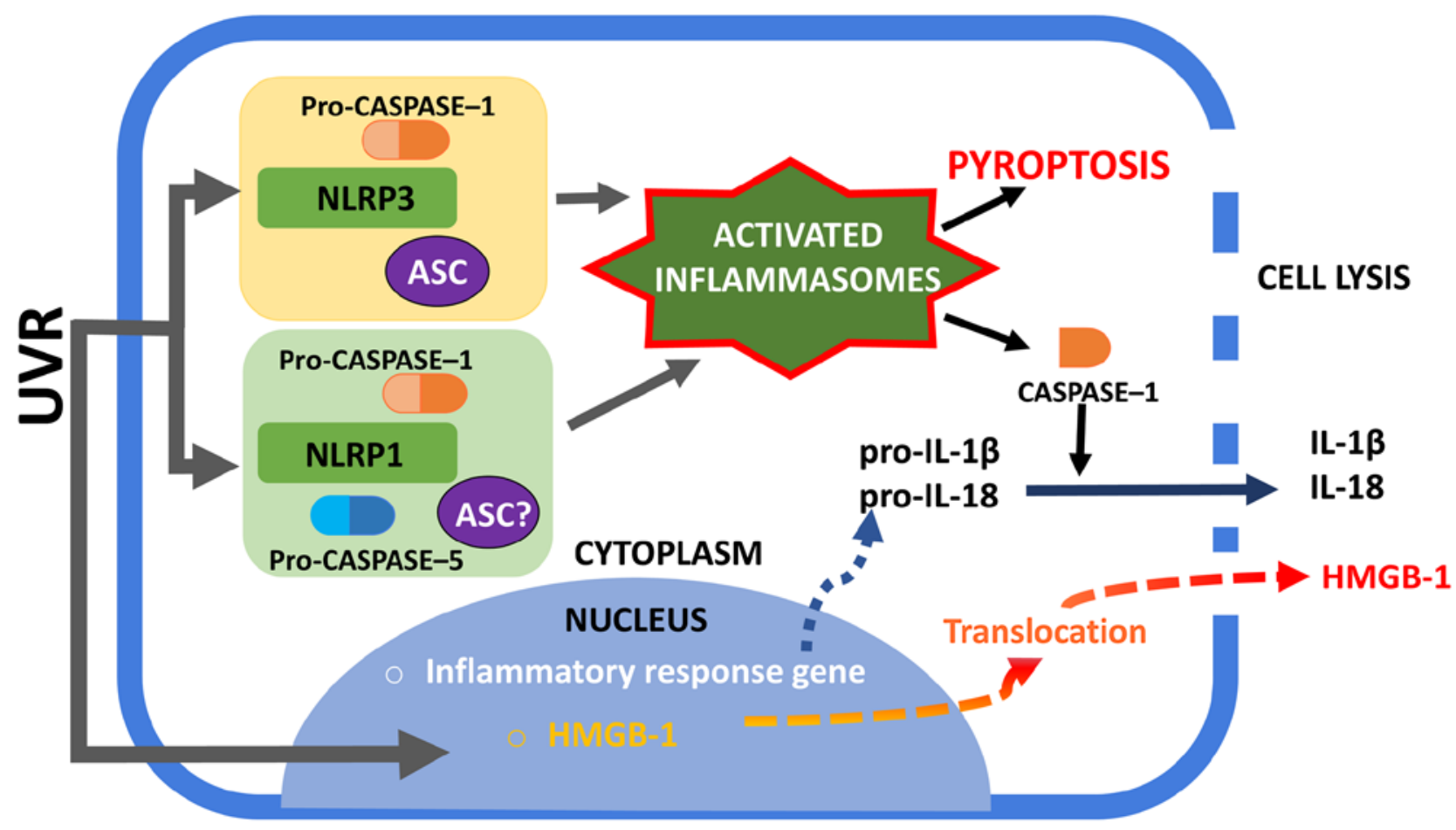

Figure 2. Activation of NLRP1 and NLRP3 inflammasomes. UVR irradiation of human keratinocytes may trigger the assembly of NLRP1 and NLRP3 inflammasomes. The NLRP1 inflammasome complex consists of caspase-1, ASC (which is not required to form complexes in murine) and NLRP1. The exact role of caspase-5 in NLRP1 inflammasome activation is unclear. The NLRP3 inflammasome is well characterized among the inflammasome complexes and consists of NLRP3, ASC and caspase-1. An active caspase- 1 form is required to process pro-IL-1 $\beta$ and pro-IL-18 into mature forms and to secrete them into the extracellular space. Furthermore, inflammasome is associated with the unconventional secretion of HMGB-1. Active caspase-1 can lead to cell pyroptosis with membrane rupture and the release of alarmins, such as HMGB1. NLRP1, NOD-like receptor family pyrin domain containing 1; NLRP3, NOD-like receptor family pyrin domain containing 3; UVR, ultraviolet radiation; ASC, apoptosis associated with Speck-like protein; HMGB-1, high mobility group box protein 1; IL, interleukin.

bind to NLRP1, thus suppressing caspase-1 activation and the secretion of Il-1 $\beta$. In some analyses, it has been shown that muramyl dipeptide (MDP) releases anti-apoptotic proteins from NLRP1, which results in inflammasome activation and the formation of oligomers (30). UVB irradiated keratinocytes show decreased levels of these anti-apoptotic proteins (Bcl-2 and $\mathrm{Bcl}-\mathrm{XL}$ ), therefore suggesting that this is a possible mechanism by which UV-induced NLRP1 activation may occur (31).

Nevertheless, Hasegawa et al (23) found that UVB-induced IL-1 $\beta$ production in human keratinocytes is selectively mediated by NLRP3, but not by NLRP1, which suggests that individual NLRPs might be specifically activated, depending on the cutaneous pathological status.

On the other hand, the results presented in 2018 by Sand et al (32) revealed that UVB irradiation activates NLRP1 inflammasomes in human primary keratinocytes, resulting in the secretion of large amounts of IL-1 $\beta$ and IL-18. Furthermore, they revealed that human fibroblasts do not secrete mature IL-1 $\beta$ or IL-18. However, these data confirm that our knowledge regarding the role of NLRP3 and NLRP1 inflammasome activation in UV-induced inflammation is still limited.

\section{NLRP3 and skin carcinogenesis}

Thus far, NLRP3 is the best described inflammasome that is involved in the pathogenesis of cryopyrin-associated peri- odic syndromes caused by mutations in the gene encoding the cytoplasmic NLRP3 associated with exacerbated IL-1 $\beta$ production (33). Other inflammatory diseases connected with increases in NLRP3 inflammasome activity that are usually associated with the polymorphism of NLRP3 have also been noted. NLRP3 inflammasomes have been implicated in the development of major diseases such as gout, type 2 diabetes, and obesity-induced insulin resistance (34). Moreover, the NLRP3 inflammasome is increasingly being suspected of playing a major role in other human pathologies such as asbestosis, Alzheimer's disease, and Parkinson's disease (35). The NLRP3 inflammasome is also an emerging key player in the development and progression of various cancers; however, its exact role in tumorigenesis remains unknown. Recent studies have shown that both the overexpression and constitutive activation of NLRP3 could contribute to the development and progression of various cancers including malignant melanoma (36), breast (5), and lung cancers (37). Furthermore, the NLRP3 inflammasome functions as a negative regulator of tumorigenesis during colitis-associated cancer (38). Thus, in those cancers NLRP3 works in conjunction with a cell or tissue.

Previous studies have indicated that the NLRP3 inflammasome together with interleukin-1 may also promote the growth of skin cancers. In studies carried out on a mouse model, Drexler et al (39) observed that mice with interleukin-1 or caspase-1 receptor knockout rarely develop dimethylbenzanthracene (DMBA) and tetradecanoylphorbolacetate (TPA)-induced skin tumors compared to wild-type mice. The 
knockout of the NLRP3 gene itself also caused a reduction in the number of skin tumors. These observations indicate that the NLRP3-dependent production of IL-1 $\beta$ may contribute to the growth of skin cancers.

Confirming this phenomenon has come from research on the adaptor protein of the NLRP3 inflammasome, ASC. The effect of ASC on several inflammasomes including NLRP3, has also been assessed in the formation of skin cancers. Deficiencies in ASC in myeloid cells in the murine model were shown to result in a significantly lower frequency of skin tumor occurrence induced by chemical agents (DMBA/TPA) (39). Interestingly, the ablation of ASC in keratinocytes in a mouse model caused the development of a greater number of tumors compared with controls. The obtained results proved that ASC suppresses tumor development in keratinocytes but promotes carcinogenesis in myeloid cells (39). Furthermore, it was found that human cutaneous squamous cell carcinoma (SCC) involves the loss of ASC protein expression.

Okamoto et al (3) produced interesting results. They showed that in cell lines derived from advanced malignant melanomas, there was a constitutive activation of the NLRP3 inflammasome which resulted in the oversecretion of IL-1 $\beta$. In cell lines derived from early forms of malignant melanoma, IL-1 $\beta$ secretion followed IL-1R activation. Furthermore, the production of IL-1 $\beta$ was inhibited by caspase- 1 suppression or IL-1R receptor blockade. As suggested by the authors, this autocrine feedback loop may be responsible for the clinical phenotype of melanoma and its progression.

In an independent study, in a model of chemical-induced carcinogenesis, Melvyn et al (40) showed that mice lacking NLRP3 have a significantly reduced tumor burden than control wild-type mice. Their data suggest that NLRP3 is an important suppressor of natural killer cell-mediated control of metastases and carcinogenesis.

\section{NLRP1 and skin carcinogenesis}

In contrast to NLRP3, NLRP1 has not been precisely analyzed. Recent studies have proven that the overexpression of NLRP1 promotes malignant melanoma progression. Zhai et al (41) found that NLRP1 can bind to caspase -2 and -9 in melanoma, thus preventing the interaction of apoptotic caspases with other activating proteins. They demonstrated that NLRP1 can negatively regulate apoptosis in malignant melanoma cells. Therefore, it is suspected that NLRP1 may inhibit the caspase-2/9-mediated apoptotic pathway. Another important link between the activation of inflammasomes and skin carcinogenesis has come from studies on polymorphisms within the gene for NLRP1 in multiple self-healing palmoplantar carcinoma (MSPC) and keratosis lichenoides chronical (KLC), whose symptoms include various ulcerative, hyperkeratotic nodular lesions on the surfaces of the hands and soles, histologically resembling well-differentiated SCC, as well as an increased predisposition to the development of SCC. Studies have shown that in both diseases, there is a gain-of-function mutation in the NLRPl gene. Under normal healthy conditions, the pyridine domain and LRR NLRP1 interact with each other, making it impossible to oligomerize the inflammasome. Mutations in the NLRPI gene cause changes in the structures of these domains, resulting in the formation of oligomers of
NLRP1 and the activation of inflammasomes. As a result, in the cell lines of keratinocytes gathered from patients suffering from MSPC and KLC, continuous production and secretion of IL-1 occurs (42). Consequently, it will be important to determine how inflammasomes drive tumor progression in future studies.

\section{Therapies that target inflammasomes}

In recent years, therapies with inflammasomes have been significantly improved to overcome human diseases such as cancer. As the activation of these multimeric cytosol complexes has been revealed in diverse human pathologies, common approaches have been set to dampen or even reduce inflammation. Most reagents that particularly target the inflammasome, caspase-1, and IL-1 have been developed and analyzed in various diseases.

Recently, much attention has been paid to the development of compounds to inhibit the IL-1 $\beta$ signaling activities as another approach for inflammasome inhibition in cancer treatment. IL-1 is a pivotal proinflammatory cytokine that stimulates angiogenesis and promotes tumor growth and metastatic dissemination, which is composed of two molecular species (IL-1 $\alpha$ and IL-1 $\beta$ ). Elaraj et al (43) revealed in the study carried out by real time quantitative reverse transcriptase PCR that several melanoma and squamous cell cancers and cell lines indicated the gene expression of IL- $1 \alpha$ and IL-1 $\beta$.

Previous studies have indicated that IL-1 also stimulates melanoma tumor growth in vivo and moreover, increased intercellular adhesion molecule-1 (ICAM-1) on the cancer cells (44). The IL-1 receptor antagonist (IL-1Ra) blocks these effects, thereby inhibiting the proliferation of skin tumor cell lines $(45,46)$, inhibiting the growth of mouse melanoma cells (47), and reducing the in vivo growth and metastatic potential of human melanoma xenografts which constitutively produce IL-1 (48). Anakinra is a recombinant human IL-1 receptor antagonist and is regarded as a biological agent that blocks the inflammatory effects of IL-1. Moreover, anti-IL-1Ra therapy significantly decreases the expression of ICAM-1 molecules. Triozzi et al (49) indicated that IL-1Ra modifies the response to macroscopic melanoma and could additionally increase the antitumor activity of the classical cytostatic chemotherapy used in melanoma treatment. As IL-1 has also been connected with the clinical toxicity of several classical chemotherapeutics used in melanoma therapy, the combination of IL-1Ra with chemotherapy has also been suggested to improve treatment outcomes (50-53). However, currently, based on the use of immunotherapy to treat melanoma, classic cytostatic agents are used very rarely and mainly in subsequent treatment lines; therefore, this ability of IL-1Ra does not actually apply. Interestingly, IL-1 has been suggested to play a crucial role in UV-induced dermal collagen degradation which is responsible for premature skin photoaging.

It has been revealed that Anakinra reduces matrix metalloproteinase-1 (MMP-1) production, which is the main enzyme that breaks down the interstitial collagen types I, II, and III, and can be used for the prevention and treatment of photoaging (54). Anakinra is currently approved and is being used clinically for the treatment of rheumatoid arthritis. What is more, this drug is currently used mostly in autoinflammatory conditions. 
NLRP3 inflammasome activation with the cleavage of caspase-1 leads to auto-inflammation through the secretion and maturation of the pro-inflammatory cytokines IL-1 $\beta$ and IL-18 in melanoma cells (55). Thymoquinone can block caspase-1 by inhibiting the NLRP3 inflammasome and thus preventing the caspase-1-induced proteolytic maturation and secretion of IL-1 $\beta$ and IL-18. It is the main bioactive constituent of the essential oil extracted from Nigella sativa seeds, which has been shown to have very promising pharmacological properties, such as antioxidant and anti-inflammatory efficacy (56-58). In many studies, it has also been reported that thymoquinone may possess anticancer effects such as the inhibition of cell proliferation, the generation of reactive oxygen species (ROS), cell cycle arrest, the induction of apoptosis, and inhibition of metastasis and angiogenesis (59). Ahmad et al (60) first demonstrated the exact antitumor function of thymoquinone and revealed that inhibition of the NLRP3 inflammasome by thymoquinone substance blocked cell migration and prevented melanoma cell metastasis in an in vivo model. These same authors also showed the inhibitory effect of metastasis through the inhibition of NLRP3, with a consequent reduction in the synthesis of IL-1 and IL-18 in an in vivo mouse model. Thymoquinone, as an antioxidant agent, may also be able to inhibit NLRP3 by reducing the production of ROS. Thus, thymoquinone has the potential to be used as a chemotherapeutic agent in combination with existing therapies for the treatment of melanoma.

Another interesting study was conducted by Ellis et al (61), who reported that physiological doses of epigallocate-chin-3-gallate, a polyphenol found in green tea, inhibits the effect of metastatic melanoma on the proliferation of human cell strains by inhibiting NLRP1, but not NLRP3 (61). Tsai et al (62) also demonstrated the role of green tea polyphenols in the inhibition of the NLRP3 inflammasome in nephritis during lupus. To the best of our knowledge, there have been no other reports on the effectiveness of other dietary agents on the reduction of metastasis by the inhibition of the NLRP3 inflammasome.

Great hope for revolutions in treatment rest on oridonin (ORI), one of the most important active Chinese medicinal components isolated from Rabdosia rubescens, which has been shown to be a potent anti-metastatic agent. Notably, oridonin shows a broad-spectrum of anti-proliferative and anti-cancer activities in various types of tumor (63-68) as well as in melanoma (69,70). Wang et al (69) indicated that ORI induces human melanoma A375-S2 cell death (69). In another independent study, Gu et al (70) reported that ORI impairs the survival capability and proliferation of melanoma cells through the induction of apoptosis (70). As the mechanisms underlying the inhibiting effects of ORI in the metastasis of melanoma cells remain unclear, many authors have investigated this topic. Li et al $(63,64)$ proposed that ORI could effectively inhibit migration as well as invasion and adhesion by inhibiting epithelial-mesenchymal transition (EMT) in melanoma cells through inhibiting the phosphorylation of phosphoiniositide-3 kinase (PI3K), Protein kinase B (Akt), and glycogen synthase kinase $3 \beta$ (GSK-3 $\beta$ ) in the presence of transforming growth factor $\beta 1$ (TGF- $\beta 1$ ). Recent studies have also indicated that oridonin is a specific inhibitor for the NLRP3 inflammasome which forms a covalent bond with the NACHT domain of NLRP3, thereby inhibiting NLRP3 inflammasome assembly and activation (71).
Despite the fact that, in recent years, great advances have been achieved in the knowledge regarding inflammasomes in cancer, there are still many questions to be answered. It is still unclear as to why inflammasomes and their components have a dual, ambivalent role in cancer development that depends on the existing tissues and the cancer stage. Moreover, the regulatory mechanisms of inflammasomes between different NLR members as well as by other signaling molecules and their interactions in tumor development are still unclear. Further studies are required to confirm the molecular mechanisms for the activation or inhibition of inflammasomes in the tumor microenvironment. Identification of the regulatory factors for the specific inflammasome may allow the determination of biomarkers for cancer prognosis as well as identifying therapeutic targets against various tumors.

There are still many knowledge gaps in the function mechanisms of inflammasomes in carcinogenesis. Better understanding of their signaling pathways and regulation may contribute to advances in human cancer prevention and treatment.

\section{Conclusions}

It is highly probable that there is a causal relationship between the activation of inflammasomes and skin carcinogenesis. However, despite many studies, this process has not been accurately described. So far, most of our knowledge about the functional importance of NLRP3 and NLRP1 inflammasomes in skin cancer has come from mouse models. Mice are nocturnal animals whose skin is protected by fur and who are only occasionally exposed to UVB radiation. This fact is particularly important when considering the profound differences between murine and human skin. Therefore, the results obtained in mouse models need to be interpreted very carefully. Furthermore, it has been revealed that the expression of inflammasome proteins is significantly lower in murine keratinocytes than in humans.

Furthermore, the effect of UV radiation on the activation of NLRP1 and NLRP3 inflammasomes has not been well researched. Some research has focused on the role of UVB in the process of inflammasome activation in keratinocytes. However, of the solar UVR reaching the surface of the Earth, about 5\% is UVB, and 95\% is UVA. Although UVA radiation is not directly absorbed by the DNA of epidermal cells, it can damage its structure through reactive oxygen species. This leads to so-called oxidative DNA damage as well as to the development of tumors. NOD-like receptors strongly react to ROS-induced cellular stress, thus resulting in the formation of inflammasomes. Cell damage by UV radiation may result in the activation of processes that are responsible for the repair of potentially damaged genetic material. By default, the mechanism of response to DNA damage (DDR) is a factor that prevents its possible transformation into cancer. Therefore, future investigation of the relationship between the UV-induced activation of inflammasome and DDR is necessary to allow for a better characterization of the processes underlying skin carcinogenesis.

The present study highlighted the recent advances in the understanding of the inflammasomes involved in skin carcinogenesis and may contribute to the development of future therapies involving the modulation of inflammasome responses for anticancer treatment. 


\section{Acknowledgements}

Not applicable.

\section{Funding}

The present study was funded by Medical University of Lodz (project no. 503/5-064-01/503-1) and The National Centre of Science (grant nos. 2017/25/N/NZ5/02064 and 2017/27/B/ NZ5/02011).

\section{Availability of data and materials}

Data sharing is not applicable to this article, as no datasets were generated or analyzed during the current study.

\section{Authors' contributions}

$\mathrm{MC}, \mathrm{IAB}$ and $\mathrm{AL}$ contributed to the conception of the study. $\mathrm{MC}$ and $\mathrm{IAB}$ performed the investigation. $\mathrm{MC}, \mathrm{IAB}$ and $\mathrm{KW}$ wrote and prepared the original draft of the manuscript. MC, JN and AL wrote, reviewed and edited the manuscript. JN and AL supervised the study.

\section{Ethics approval and consent to participate}

Not applicable.

\section{Patient consent for publication}

Not applicable.

\section{Competing interests}

The authors declare that they have no competing interests.

\section{References}

1. Hanahan D and Weinberg RA: Hallmarks of cancer: The next generation. Cell 144: 646-674, 2011.

2. Kantono $M$ and Guo B: Inflammasomes and cancer: The dynamic role of the inflammasome in tumor development. Front Immunol 8: 1132, 2017.

3. Okamoto M, Liu W, Luo Y, Tanaka A, Cai X, Norris DA, Dinarello CA and Fujita M: Constitutively active inflammasome in human melanoma cells mediating autoinflammation via caspase-1 processing and secretion of interleukin-1beta. J Biol Chem 285: 6477-6488, 2010.

4. Nasti TH and Timares L: Inflammasome activation of IL-1 family mediators in response to cutaneous photodamage. Photochem Photobiol 88: 1111-1125, 2012.

5. Rathinam VAK and Fitzgerald KA: Inflammasome complexes: Emerging mechanisms and effector functions. Cell 165: 792-800, 2016.

6. de Zoete MR, Palm NW, Zhu S and Flavell RA: Inflammasomes. Cold Spring Harb Perspect Biol 6: a016287, 2014.

7. Lamkanfi M: Emerging inflammasome effector mechanisms. Nat Rev Immunol 11: 213-220, 2011.

8. Martinon F, Burns K and Tschopp J: The inflammasome: A molecular platform triggering activation of inflammatory caspases and processing of proIL-beta. Mol Cell 10: 417-426, 2002.

9. Frew BC, Joag VR and Mogridge J: Proteolytic processing of Nlrplb is required for inflammasome activity. PLoS Pathog 8: e1002659, 2012

10. Nour AM, Yeung YG, Santambrogio L, Boyden ED, Stanley ER and Brojatsch J: Anthrax lethal toxin triggers the formation of a membrane-associated inflammasome complex in murine macrophages. Infect Immun 77: 1262-1271, 2009.
11. Faustin B, Lartigue L, Bruey JM, Luciano F, Sergienko E, Bailly-Maitre B, Volkmann N, Hanein D, Rouiller I and Reed JC: Reconstituted NALP1 inflammasome reveals two-step mechanism of caspase-1 activation. Mol Cell 25: 713-724, 2007.

12. Van Opdenbosch N, Gurung P, Vande Walle L, Fossoul A, Kanneganti TD and Lamkanfi M: Activation of the NLRP1b inflammasome independently of ASC-mediated caspase-1 autoproteolysis and speck formation. Nat Commun 5: 3209, 2014.

13. Guey B, Bodnar M, Manié SN, Tardivel A and Petrilli V: Caspase-1 autoproteolysis is differentially required for NLRP1b and NLRP3 inflammasome function. Proc Natl Acad Sci USA 111: 17254-17259, 2014.

14. Bauernfeind $\mathrm{F}$ and Ablasser A: Inflammasomes: Current understanding and open questions. Cell Mol Life Sci 68: 765-783, 2011.

15. Finger JN, Lich JD, Dare LC, Cook MN, Brown KK, Duraiswami C, Bertin J and Gough PJ: Autolytic proteolysis within the function to find domain (FIIND) is required for NLRP1 inflammasome activity. J Biol Chem 287: 25030-25037, 2012.

16. Yu CH, Moecking J, Geyer M and Masters SL: Mechanisms of NLRP1 mediated autoinflammatory disease in humans and mice. J Mol Biol 430: 142-152, 2018.

17. Lamkanfi $M$ and Dixit VM: Mechanisms and functions of inflammasomes. Cell 157: 1013-1022, 2014.

18. Kayagaki N, Warming S, Lamkanfi M, Vande Walle L, Louie S, Dong J, Newton K, Qu Y, Liu J, Heldens S, et al: Non-canonical inflammasome activation targets caspase-11. Nature 479: 117-121, 2011.

19. Shi J, Zhao Y, Wang Y, Gao W, Ding J, Li P, Hu L and Shao F: Inflammatory caspases are innate immune receptors for intracellular LPS. Nature 514: 187-192, 2014.

20. Liu $X$ and Lieberman J: A mechanistic understanding of pyroptosis: The fiery death triggered by invasive infection. Adv Immunol 135: 81-117, 2017.

21. Zhao D, Wu Y, Zhuang J, Xu C and Zhang F: Activation of NLRP1 and NLRP3 inflammasomes contributed to cyclic stretch-induced pyroptosis and release of IL-1 $\beta$ in human periodontal ligament cells. Oncotarget 7: 68292-68302, 2016.

22. Feldmeyer L, Keller M, Niklaus G, Hohl D, Werner S and Beer HD: The inflammasome mediates UVB-induced activation and secretion of interleukin-1beta by keratinocytes. Curr Biol 17: 1140-1145, 2007.

23. Hasegawa T, Nakashima $M$ and Suzuki Y: Nuclear DNA damage-triggered NLRP3 inflammasome activation promotes UVB-induced inflammatory responses in human keratinocytes. Biochem Biophys Res Commun 477: 329-335, 2016.

24. Strittmatter GE, Sand J, Sauter M, Seyffert M, Steigerwald R, Fraefel C, Smola S, French LE and Beer HD: IFN- $\gamma$ primes keratinocytes for HSV-1-induced inflammasome activation. J Invest Dermatol 136: 610-620, 2016.

25. Reinholz M, Kawakami Y, Salzer S, Kreuter A, Dombrowski Y, Koglin S, Kresse S, Ruzicka T and Schauber J: HPV16 activates the AIM2 inflammasome in keratinocytes. Arch Dermatol Res 305: 723-732, 2013.

26. Ekman AK, Verma D, Fredrikson M, Bivik C and Enerbäck C: Genetic variations of NLRP1: susceptibility in psoriasis. $\mathrm{Br}$ J Dermatol 171: 1517-1520, 2014.

27. Bivik C, Verma D, Winge MC, Lieden A, Bradley M, Rosdahl I and Söderkvist P: Genetic variation in the inflammasome and atopic dermatitis susceptibility. J Invest Dermatol 133: 2486-2489, 2013.

28. Levandowski CB, Mailloux CM, Ferrara TM, Gowan K, Ben S, Jin Y, McFann KK, Holland PJ, Fain PR, Dinarello CA and Spritz RA: NLRP1 haplo-types associated with vitiligo and autoimmunity increase interleukin-1 $\beta$ processing via the NLRP1 inflammasome. Proc Natl Acad Sci USA 110: 2952e2956, 2013.

29. Faustin B and Reed JC: Sunburned skin activates inflammasomes. Trends Cell Biol 18: 4-8, 2008.

30. Faustin B, Chen Y, Zhai D, Le Negrate G, Lartigue L, Satterthwait A and Reed JC: Mechanism of Bcl-2 and Bcl-X(L) inhibition of NLRP1 inflammasome: Loop domain-dependent suppression of ATP binding and oligomerization. Proc Natl Acad Sci USA 106: 3935-3940, 2009.

31. Reagan-Shaw S, Breur J and Ahmad N: Enhancement of UVB radiation-mediated apoptosis by sanguinarine in HaCaT human immortalized keratinocytes. Mol Cancer Ther 5: 418-429, 2006.

32. Sand J, Haertel E, Biedermann T, Contassot E, Reichmann E, French LE, Werner S and Beer HD: Expression of inflammasome proteins and inflammasome activation occurs in human, but not in murine keratinocytes. Cell Death Dis 9: 24, 2018. 
33. Gurung P and Kanneganti TD: Autoinflammatory skin disorders: The inflammasome in focus. Trends Mol Med 22: 545-564, 2016.

34. Liu-Bryan R: Intracellular innate immunity in gouty arthritis: Role of NALP3 inflammasome. Immunol Cell Biol 88: 20-23, 2010

35. Shi F, Kouadir M and Yang Y: NALP3 inflammasome activation in protein misfolding diseases. Life Sci 135: 9-14, 2015.

36. Liu W, Luo Y, Dunn JH, Norris DA, Dinarello CA and Fujita M: Dual role of apoptosis-associated speck-like protein containing a CARD (ASC) in tumorigenesis of human melanoma. J Invest Dermatol 133: 518-527, 2013

37. Wang Y, Kong H, Zeng X, Liu W, Wang Z, Yan X, Wang H and Xie W: Activation of NLRP3 inflammasome enhances the proliferation and migration of A549 lung cancer cells. Oncol Rep 35 2053-2064, 2016.

38. Allen IC, TeKippe EM, Woodford RM, Uronis JM, Holl EK, Rogers $\mathrm{AB}$, Herfarth $\mathrm{HH}$, Jobin $\mathrm{C}$ and Ting JP: The NLRP3 inflammasome functions as a negative regulator of tumorigenesis during colitis-associated cancer. J Exp Med 207: 1045-1056, 2010.

39. Drexler SK, Bonsignore L, Masin M, Tardivel A, Jackstadt R, Hermeking H, Schneider P, Gross O, Tschopp J and Yazdi AS: Tissue-specific opposing functions of the inflammasome adaptor ASC in the regulation of epithelial skin carcinogenesis. Proc Natl Acad Sci 109: 18384-18389, 2012.

40. Melvin JC, Holmberg L, Rohrmann S, Loda M and Van Hemelrijck M: Serum lipid profiles and cancer risk in the context of obesity: Four meta-analyses. J Cancer Epidemiol 2013: 823849, 2013.

41. Zhai Z, Liu W, Kaur M, Luo Y, Domenico J, Samson JM, Shellman YG, Norris DA, Dinarello CA, Spritz RA and Fujita M: NLRP1 promotes tumor growth by enhancing inflammasome activation and suppressing apoptosis in metastatic melanoma. Oncogene 36: 3820-3830, 2017.

42. Zhong FL, Mamaï O, Sborgi L, Boussofara L, Hopkins R, Robinson K, Szeverényi I, Takeichi T, Balaji R, Lau A, et al: Germline NLRP1 mutations cause skin inflammatory and cancer susceptibility syndromes via inflammasome activation. Cell 167 187-202.e17, 2016

43. Elaraj DM, Weinreich DM, Varghese S, Puhlmann M, Hewitt SM, Carroll SM, Feldman ED, Turner EM and Alexander HR: The role of interleukin 1 in growth and metastasis of human cancer xenografts. Clin Cancer Res 12: 1088-1096, 2006.

44. Pazyar N, Feily A and Yaghoobi R: An overview of interleukin-1 receptor antagonist, anakinra, in the treatment of cutaneous diseases. Curr Clin Pharmacol 7: 271-275, 2012.

45. LaE, Rundhaug JE and Fischer SM: Role of intracellular interleukin-1 antagonist in skin carcinogenesis. Mol Carcinog 30: 218-223, 2001.

46. Bar D, Apte RN, Voronov E, Dinarello CA and Cohen S: A continuous delivery system of IL-1 receptor antagonist reduces angiogenesis and inhibits tumor development. FASEB J 18 : 161-163, 2004

47. McKenzie RC, Oran A, Dinarello CA and Sauder DN: Interleukin-1 receptor antagonist inhibits subcutaneous B 16 melanoma growth in vivo. Anticancer Res 16: 437-441, 1996.

48. Weinreich DM, Elaraj DM, Puhlmann M, Hewitt SM, Carroll NM, Feldman ED, Turner EM, Spiess PJ and Alexander HR: Effect of interleukin 1 receptor antagonist gene transduction on human melanoma xenografts in nude mice. Cancer Res 63: 5957-5961, 2003.

49. Triozzi PL and Aldrich W: Effects of interleukin-1 receptor antagonist and chemotherapy on host-tumor interactions in established melanoma. Anticancer Res 30: 345-354, 2010

50. Hoshino T, Okamoto M, Sakazaki Y, Kato S, Young HA and Aizawa H: Role of proinflammatory cytokine IL-18 and IL-1beta in bleomycin-induced lung injury in humans and mice. Am J Respir Cell Mol Biol 41: 661-670, 2009.

51. Ledeboer A, Jekich BM, Sloane EM, Mahoney JH, Langer SJ, Milligan ED, Martin D, Maier SF, Johnson KW, Leinwand LA, et al: Intrathecal interleukin-10 gene therapy attenuates paclitaxel-induced mechanical allodynia and proinflammatory cytokine expression in dorsal root ganglia in rats. Brain Behav Immun 21: 686-698, 2007.

52. Twining CM, Sloane EM, Milligan ED, Chacur M, Martin D, Poole S, Marsh H, Maier SF and Watkins LR: Peri-sciatic proinflammatory cytokines, reactive oxygen species, and complement induce mirror-image neuropathic pain in rats. Pain 110: 299-309, 2004.

53. Surguladze D, Deevi D, Claros N, Corcoran E, Wang W, Plym MJ, Wu Y, Doody J, Mauro DJ, Witte L, et al: Tumor necrosis factor-alpha and interleukin-1 antagonists alleviate inflammatory skin changes associated with epidermal growth factor receptor antibody therapy in mice. Cancer Res 69: 5643-5647, 2009.
54. Wang X, Bi Z, Chu W and Wan Y: IL-1 receptor antagonist attenuates MAP kinase/AP-1 activation and MMP1 expression in UVA-irradiated human fibroblasts induced by culture medium from UVB-irradiated human skin keratinocytes. Int J Mol Med 16: 1117-1124, 2005

55. van de Veerdonk FL, Netea MG, Dinarello CA and Joosten LA: Inflammasome activation and IL-1 $\beta$ and IL-18 processing during infection. Trends Immunol 32: 110-116, 2011

56. Abdelmeguid NE, Fakhoury R, Kamal SM and Al Wafai RJ: Effects of Nigella sativa and thymoquinone on biochemical and subcellular changes in pancreatic $\beta$-cells of streptozotocin-induced diabetic rats. J Diabetes 2: 256-266, 2010.

57. Kanter M: Nigella sativa and derived thymoquinone prevents hippocampal neurodegeneration after chronic toluene exposure in rats. Neurochem Res 33: 579-588, 2008.

58. Woo CC, Kumar AP, Sethi G and Tan KH: Thymoquinone: Potential cure for inflammatory disorders and cancer. Biochem Pharmacol 83: 443-451, 2012

59. Banerjee S, Padhye S, Azmi A, Wang Z, Philip PA, Kucuk O, Sarkar FH and Mohammad RM: Review on molecular and therapeutic potential of thymoquinone in cancer. Nutr Cancer 62: 938-946, 2010

60. Ahmad I, Muneer KM, Tamimi IA, Chang ME, Ata MO and Yusuf N: Thymoquinone suppresses metastasis of melanoma cells by inhibition of NLRP3 inflammasome. Toxicol Appl Pharmacol 270: 70-76, 2013.

61. Ellis LZ, Liu W, Luo Y, Okamoto M, Qu D, Dunn JH and Fujita M: Green tea polyphenol epigallocatechin-3-gallate suppresses melanoma growth by inhibiting inflammasome and IL-1 $\beta$ secretion. Biochem Biophys Res Commun 414: 551-556, 2011.

62. Tsai PY, Ka SM, Chang JM, Chen HC, Shui HA, Li CY, Hua KF, Chang WL, Huang JJ, Yang SS and Chen A: Epigallocatechin-3-gallate prevents lupus nephritis development in mice via enhancing the Nrf2 antioxidant pathway and inhibiting NLRP3 inflammasome activation. Free Radic Biol Med 51: 744-754, 2011.

63. Li D, Han T, Liao J, Hu X, Xu S, Tian K, Gu X, Cheng K, Li Z $\mathrm{Hua} \mathrm{H}$ and $\mathrm{Xu} \mathrm{J}$ : Oridonin, a promising ent-Kaurane diterpenoid lead compound. Int J Mol Sci 17: E1395, 2016.

64. Li D, Han T, Xu S, Zhou T, Tian K, Hu X, Cheng K, Li Z, Hua H and $\mathrm{Xu} \mathrm{J}$ : Antitumor and antibacterial derivatives of oridonin: A main composition of Dong-Ling-Cao. Molecules 21: E575, 2016.

65. Lu J, Chen X, Qu S, Yao B, Xu Y, Wu J, Jin Y and Ma C: Oridonin induces $G_{2} / M$ cell cycle arrest and apoptosis via the PI3K/Akt signaling pathway in hormone-independent prostate cancer cells. Oncol Lett 13: 2838-2846, 2017.

66. Wang XH, Zhang SF, Bao JT and Liu FY: Oridonin synergizes with Nutlin-3 in osteosarcoma cells by modulating the levels of multiple Bcl-2 family proteins. Tumour Biol 39: 1010428317701638, 2017.

67. Xia S,Zhang X, Li C and Guan H: Oridonin inhibits breast cancer growth and metastasis through blocking the Notch signaling. Saudi Pharm J 25: 638-643, 2017.

68. Zhang Y, Wang L, Zi Y, Zhang L, Guo Y and Huang Y: Oridonin effectively reverses the drug resistance of cisplatin involving induction of cell apoptosis and inhibition of MMP expression in human acute myeloid leukemia cells. Saudi J Biol Sci 24: 678-686, 2017.

69. Wang HJ, Li D, Yang FY, Tashiro S, Onodera S and Ikejima T: Oridonin induces human melanoma A375-S2 cell death partially through inhibiting insulin-like growth factor 1 receptor signaling. J Asian Nat Prod Res 10: 787-798, 2008.

70. Gu Z, Wang X, Qi R, Wei L, Huo Y, Ma Y, Shi L, Chang Y, $\mathrm{Li}$ and Zhou L: Oridonin induces apoptosis in uveal melanoma cells by upregulation of Bim and downregulation of fatty acid synthase. Biochem Biophys Res Commun 457: 187-193, 2015.

71. He H and Jiang Y: Oridonin is a covalent NLRP3 inhibitor with strong anti-inflammasome activity. Nat Commun 9: 2550, 2018.

This work is licensed under a Creative Commons Attribution-NonCommercial-NoDerivatives 4.0 International (CC BY-NC-ND 4.0) License. 